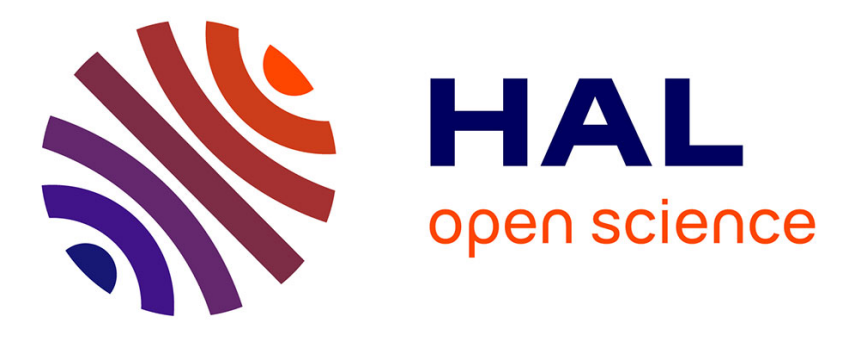

\title{
Use of Plastic Optical Fibers for Distributed MIMO in Li-Fi Systems
}

Sepideh Mohammadi Kouhini, Elnaz Alizadeh Jarchlo, Ricardo Pinto Ferreira, Sasan Khademi, Gerhard Maierbacher, Bernhard Siessegger, Dominic Schulz, Jonas Hilt, Peter Hellwig, Volker Jungnickel

\section{To cite this version:}

Sepideh Mohammadi Kouhini, Elnaz Alizadeh Jarchlo, Ricardo Pinto Ferreira, Sasan Khademi, Gerhard Maierbacher, et al.. Use of Plastic Optical Fibers for Distributed MIMO in Li-Fi Systems. 2019 Global LIFI Congress (GLC), Jun 2019, Paris, France. 10.1109/GLC.2019.8864130 . hal-02563771

\section{HAL Id: hal-02563771 https://hal.science/hal-02563771}

Submitted on 5 May 2020

HAL is a multi-disciplinary open access archive for the deposit and dissemination of scientific research documents, whether they are published or not. The documents may come from teaching and research institutions in France or abroad, or from public or private research centers.
L'archive ouverte pluridisciplinaire HAL, est destinée au dépôt et à la diffusion de documents scientifiques de niveau recherche, publiés ou non, émanant des établissements d'enseignement et de recherche français ou étrangers, des laboratoires publics ou privés. 


\title{
Use of Plastic Optical Fibers for Distributed MIMO in Li-Fi Systems
}

\author{
Sepideh Mohammadi Kouhini ${ }^{1}$, Elnaz Alizadeh Jarchlo ${ }^{1}$, Ricardo Ferreira ${ }^{1}$, Sasan Khademi, Gerhard Maierbacher ${ }^{1}$, \\ Bernhard Siessegger ${ }^{1}$, Dominic Schulz ${ }^{2}$, Jonas Hilt ${ }^{2}$, Peter Hellwig ${ }^{2}$, Volker Jungnickel ${ }^{2}$ \\ ${ }^{1}$ OSRAM GmbH, 85748 Garching, Germany, ${ }^{2}$ Fraunhofer HHI, 10587 Berlin, Germany. \\ S.Kouhini@osram.com, Volker.jungnickel@hhi.fraunhofer.de
}

\begin{abstract}
Previous researches on optical wireless communication (OWC) focused mainly on increasing the data rate for mobile broadband delivery. However, for new applications such as industrial wireless, reliability and robustness against interference play a crucial role. This paper focuses on the design, characterization, and real-world testing of novel solutions for OWC taking industrial requirements into account. Recent experimental works have demonstrated the feasibility of reliable OWC in a manufacturing environment. Here we propose to implement networked OWC which is also known as $\mathrm{Li}-\mathrm{Fi}$ by means of a distributed MIMO approach enabling ultra-reliable low-latency communication (URLLC) which is an important use case for 5G and beyond mobile networks. For distributing the MIMO signals, plastic optic fiber (POF) is a promising low-cost solution offering high data rates, easy deployment and inherent robustness against electromagnetic interference. As POF may become a main component for Li-Fi using distributed MIMO, commercially available POF solutions are studied and their usability for this new application to distribute signal between central unit and multiple frontends is discussed.
\end{abstract}

Index Terms-Plastic optical fiber, Optical Wireless Communication, MIMO.

\section{INTRODUCTION}

Optical Wireless Communication (OWC) is a promising area of research with the potential to provide reliable mobile communication in industrial scenarios [1]. OWC allows access to a huge amount of unregulated bandwidth in the optical spectrum without interfering with radio frequency (RF) based systems. Further benefits include secure communication due to spatial confinement of communication inside the light cone, robustness against electromagnetic interference (EMI), jamming, and reuse of the existing illumination infrastructure.

A new application of OWC is wireless connectivity in manufacturing environments [2]. The specific requirements for mobile communication in this scenario are: high level of security, robustness against EMI, reliable communication with moderate data rates (up to $100 \mathrm{Mbit} / \mathrm{s}$ with the potential of Gbit/s) and low latency (few milliseconds) [4]. OWC is a good candidate to serve these industrial requirements. However, OWC links depend strongly on the line of sight (LOS), as even first-order reflections are $20 \mathrm{~dB}$ and more in the electrical domain below the LOS signal. Thus, OWC links are easily broken by shadowing or blocking from standing or moving objects.

One way to overcome blocking is multiple-input multipleoutput (MIMO). MIMO is state-of-the-art in Wi-Fi and

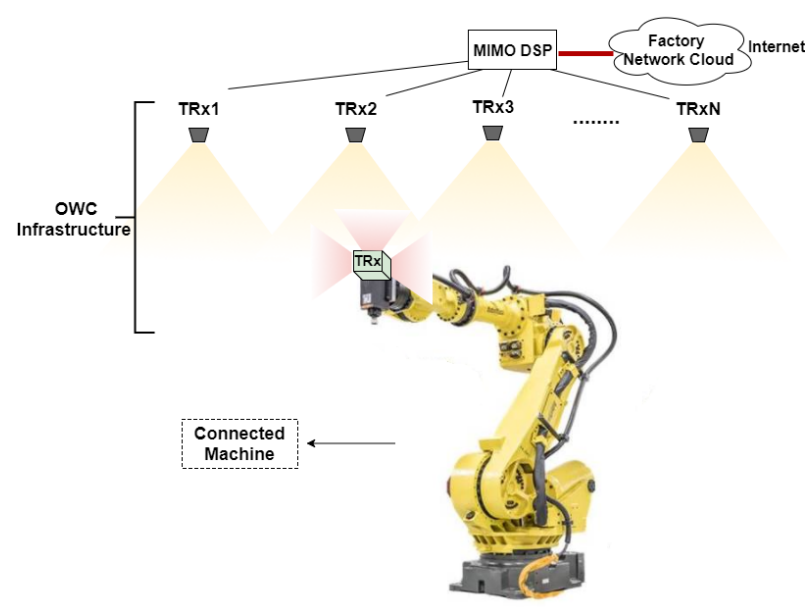

Fig. 1: Example of a distributed MIMO system for reliable OWC between the factory network and a mobile robot. The robot is equipped with an OWC unit providing omnidirectional coverage. Multiple optical front-ends (OFE) are arranged at the ceiling, like in an illumination infrastructure, and provide cohesive and overlapping coverage. A centralized digital signal processor (DSP) is connected to the distributed OFEs via fixed links to enable MIMO communication.The DSP is connected to the factory network.

modern cellular networks [5] and it can improve both, data rate and reliability of wireless links [7]. On the one hand, MIMO can be used to multiply the capacity of a wireless link by using transmitting parallel data streams and exploit the multi-path nature of wireless propagation in this way. This is known as spatial multiplexing [6]. On the other hand, MIMO can be used to overcome detrimental effects due to blocking and multi-path fading. Spatial diversity is implemented by sending and receiving the same data from multiple transmitters and receivers, respectively. There is a fundamental trade-off between multiplexing and diversity. In a distributed MIMO (D-MIMO) system, multiple optical front-end (OFE) units are deployed in distributed manner to achieve homogeneous coverage in the intended area. In this way, the mobile device can be connected to multiple OFEs, what will be essential for high availability [8]. On the other hand transmitted and received signals are fed from and into the joint DSP. D-MIMO is a new form of implementing networked OWC systems also denoted as Li-Fi. 
A recent proof-of-concept explored D-MIMO for OWC in a car manufacturing cell at BMW's robotics test lab [4]. Ana$\log$ OWC baseband signals were transmitted over twistedpair cables usually used for Ethernet. Above $70 \mathrm{MHz}$, EMI was observed due to FM radio broadcast from a nearby TV tower. Unintentionally, the long cables acted as antennas [4]. For connecting the central DSP in Fig. 1 to the OFEs, there are several other fixed home networking technologies. These are power line communications (PLC), coax cable and plastic optical fiber (POF). OWC is frequently regarded as an extension of these technologies into the wireless domain. In this paper, the use of POF is studied for distributing OWC signals over short indoor distances between centralized DSP and distributed OFEs, similar to the well-known radio-overfiber (RoF) concept [10]. POF is used as an optical relay technology being simple, low-cost and inherently robust against EMI like OWC.

The paper is organized as follows: Section II highlights the challenges of OWC in industrial scenario and proposes distributed MIMO as potential solution. In Section III, a proofof-concept implementation is presented. Section IV reports our experimental results. Section V provides a summary and outlook of future research.

\section{Distributed MIMO FOR OWC IN INDUSTRY}

In an industrial manufacturing process, any interruption can cause a detrimental economic damage to the manufacturer. For instance, in automotive manufacturing, one vehicle is produced per minute nowadays. Stopping the production line for one hour can cause a loss of several Million Euros. Therefore, reliable communication plays a vital role in industry.

In industrial use cases with high mobility, ensuring reliability is often not easily doable. For instance, in robot manufacturing as demonstrated in Fig. 1, the robot's arm moves and rotates fast in 3D space so that the LOS can be blocked rapidly in 10 milliseconds. D-MIMO can then be used to overcome blockages and provide reliable connectivity. While one link can be broken, this is unlikely for multiple links in a distributed MIMO setup [4]. Therefore, D-MIMO is proposed as an efficient solution to overcome the corresponding signal fades of 20 to $30 \mathrm{~dB}$ in the electrical domain during sudden movements and rotations [4]. However, using twisted pair for distributing OWC signals needs a considerable effort in terms of shielding, interconnect and possibly special cables in order not to be sensitive to EMI. As EMI is regarded critical for industry scenarios, POF is considered as reliable low-cost solution to distribute the MIMO signals to optical front-ends.

\section{A. POF-based transmission of OWC signals}

A main disadvantage of electrical cables in manufacturing is robustness in harsh EMI [9]. Optical communication is inherently robust and offers high data rates [11], [12]. In contrast to glass fibers, being widely used as backbone in data centers, local and wide area networks, polymer fibers are mechanically robust, lightweight and have potentially lower

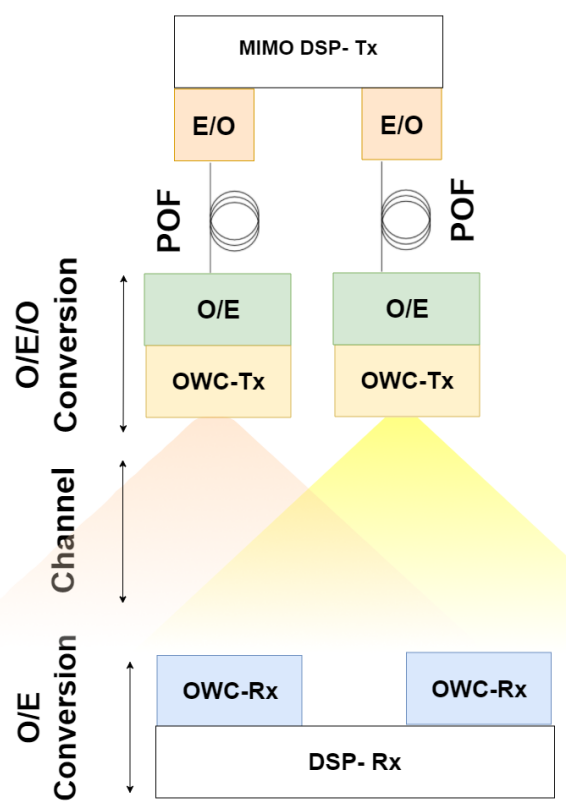

Fig. 2: In this paper, analog transmission of OWC signals over POF is investigated. The diagram shows the downlink only for simplicity, the uplink works similarly.

cost. POFs facilitate fast installation, flexible adaption and simple expansion using standardized connectors. A major reason for low cost is the relatively large core diameter of the POF, which is in the order of one millimeter. It allows efficient coupling of visible light into and out of the POF from LEDs and to large-area silicon photodiodes (LA-PDs), respectively. In fact, LEDs and LA-PDs are fast enough for current industrial applications. Fast modulation requires sophisticated LED drivers and trans-impedance amplifiers. Both aim at ideal impedance matching similar to OWC.

\section{B. Analog D-MIMO over POF Concept}

Figure 2 presents the D-MIMO system concept for transmission of OWC signals over POF which is operated as a relay link. Relays can be operated in two modes: amplifyand-forward (A\&F) and decode-and-forward (D\&F). In this paper, only the A\&F mode is considered where analog baseband signals are transmitted in a transparent manner between DSP and OFE. This scheme is favorable for low latency and it needs no additional synchronization effort but may have implications on the performance.

In Figure 2, data is passed into a MIMO DSP generating the OWC waveform. Next the waveform is transmitted over POF. Therefore, the signal is passed through the electricalto-optical converter (E/O) block, the modulated light then passes through the POF, which can be up to several ten meters long and introduce multi-path due to modal dispersion in the fiber, before being received at the optical-to-electrical converter $(\mathrm{O} / \mathrm{E})$ where a small photocurrent is detected 
proportional to the received optical signal power. This current is amplified and used to directly modulate the OWC-Tx whose signal is transmitted over the optical wireless channel. At the OWC-Rx, the signal is retrieved from channel and amplified. Due to light propagation in free space, the optical signal can be attenuated and received via multi-paths. The demodulation process and data recovery is finally operated in the DSP-Rx where the received signal is impaired by thermal noise and bandwidth limitation of the POF link before it is directly used as a baseband signal in the OFE for the OWC link. OFEs and transmission over the wireless channel add further distortion.

Accordingly, the signal is distorted twice, and the compound channel is used as a single effective link for communication. ${ }^{1}$

\section{PROOF-OF-CONCEPT}

First, a proof-of-concept for the POF-based OWC link has been setup in the lab. It consists of analog transmission through a POF link followed by an OWC link.

\section{A. POF-Link}

The schematic implemented for the POF transmission is shown in figure 3. Electrical-to-optical and the optical-toelectrical conversion is preformed inside the LED driver and receiver units section. The transmitter contains a 650 $\mathrm{nm}$ LED, which is driven by a high bandwidth driver at frequencies up to $250 \mathrm{MHz}$. It converts the input voltage linearly into an output current for the LED. Communication works via modulating the signal onto the LED using intensity modulation (IM) of the LED light [13]. The light is passed through a step-index POF as a transmission medium with various lengths. The receiver detects the light by direct detection (DD) [13] using an integrated fiber-optical receiver by using a large-area (LA) photodiode, together with the trans-impedance amplifier, and provides linear conversion of modulated light into a differential analog electrical signal. The TIA used with detector must provide low noise over the intended signal bandwidth above $150 \mathrm{MHz}$ same as the OWC units used in this paper. Therefore, the TIA must be designed properly to take the capacitance and current characteristics of the detector into account. Within the design procedure of the TIA, dynamic range, cut-off frequency, temperature stability and isolation from extraneous noise have been taken into account. The amplifier's feedback loop must be stabilized by a suitable phase margin compensation and sufficient gain. On the other hand, choosing the TIA feedback causes tradeoff between gain and useful bandwidth. In addition, the DC offset cancellation method has been used to avoid output saturation when connecting POF Link to the OWC units.

\section{B. OWC link}

Figure 4 shows two OWC units capable of real-time optical communication at around $100 \mathrm{Mbit} / \mathrm{s}$ over a distance of $10 \mathrm{~m}$. The OWC units have been developed in

\footnotetext{
${ }^{1}$ This is in contrast to the D\&F relay where the received signal is first decoded and corrected for any errors before digital data are transmitted using a second waveform over the second link.
}

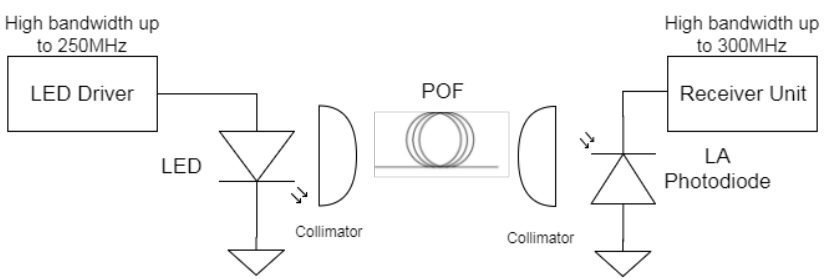

Fig. 3: Block diagram of setup to establish the POF link. It shows the stages for LED driver, POF and receiver units.

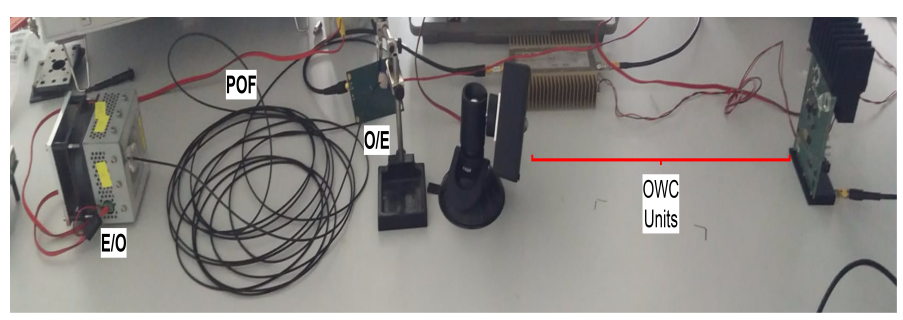

Fig. 4: Hardware implementation of POF and OWC link including POF AFEs and two OWC units with a point-topoint configuration is shown. The POF link has 10 meters length and both OWC units are configurable to act as an access point or as a client which, can operate for distances over $10 \mathrm{~m}$.

a separate project. They consist of a DSP using orthogonal frequency-division multiplex (OFDM) over $100 \mathrm{MHz}$ bandwidth with closed-loop adaptive bitloading to address frequency-selective channel characteristics. All digital signal processing follows ITU-T recommendation G.hn-2011 in the coax mode. The analog board is custom-designed to allow high optical transmit power and high sensitivity using offthe-shelf high-power LEDs operating at $860 \mathrm{~nm}$ (OSRAM SFH 4715 AS) and large-area silicon photodiodes (PD, Hamamatsu S6968).

The driver performs impedance matching for 4 LEDs operated in parallel producing altogether 2.5 Watts of average optical power emitted into a beam width of $90^{\circ}$ full width at half maximum (FWHM). 5 PDs with individual TIAs and equal gain combining are used to capture enough light. The LED driver uses a fixed bias current while the timevarying modulation current for the data signal is optimized to maximize data rate at the intended working distance. ${ }^{2}$ Various orientations of the 5 individual PDs with respect to the optical axis are supported in order to achieve a wider field-of-view (FOV) of $\approx 90^{\circ}$ FWHM.

\section{RESULTS AND DisCUSSION}

Next, the analog signal path has been characterized.

\section{A. Analog characterization}

This section describes analog tests of POF and OWC links.

${ }^{2}$ When modulation amplitude is increased, at first, more signal is received and data rate is increased. At a certain modulation amplitude, however, the OFDM waveform gets clipped and data rate will be reduced, accordingly. The optimal modulation current is found easily by maximizing the data rate. 


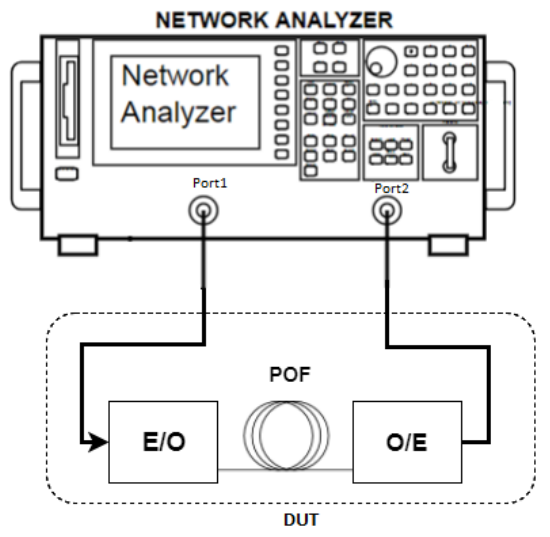

Fig. 5: Setup to measure the frequency response of a POF link using a vector network analyzer (VNA).

\section{1) POF Link}

Frequency response and dynamic range have been measured over the end-to-end POF link as shown in Figure 5. The vector network analyzer (VNA) works as both, signal source and analyzer. It provides a sine wave like a function generator whose frequency is swept over time and measures at each frequency the received amplitude and phase. In order to evaluate the performance of a LED, a high bandwidth photo receiver has been used for signal recovery. In this way, pure bandwidth of the driver and LED is estimated as shown in figure 6 .

In the next step, the POF is connected to the PD to observe the performance of the LED and POF together as illustrated in figure 6. In general, bandwidth of POF depends on the length of the fiber due to modal dispersion [15]. For short distance, bandwidth is not significantly changed up to typical indoor distance up to 10 meters, see Fig. 6. However, at frequencies above $100 \mathrm{MHz}$, the amplitude response shows a higher attenuation including a notch attributed to modal dispersion in the POF [15]. Although the notch costs a fraction of the useful bandwidth, it is narrow compared to the overall useful bandwidth. Adaptive OFDM will use both, the bandwidth below and above the notch so it will not significantly reduce the POF link performance.

\section{2) Analog $P O F+O W C$ link}

The analog link consists of the combination of the POF and OWC links as shown in figure 4 and 7 . The frequency response of OWC link is measured in a similar way like in the previous subsection and shown in figure 5 by using the OWC units. The blue line in figure 8 shows the measured frequency response for the OWC link with a $-3 \mathrm{~dB}$ and -10 $\mathrm{dB}$ bandwidth of around 105 and $167 \mathrm{MHz}$, respectively. The analog $\mathrm{POF}+\mathrm{OWC}$ link is also shown in figure 8 by the yellow line with a $-3 \mathrm{~dB}$ and $-10 \mathrm{~dB}$ bandwidth of $71 \mathrm{MHz}$ and $122 \mathrm{MHz}$ for the POF with length of 10 meter, respectively. This indicates that the bandwidth of the $\mathrm{POF}+\mathrm{OWC}$ link is sufficient for the manufacturing scenario which requires moderate data rates no more than $100 \mathrm{Mbit} / \mathrm{s}$.

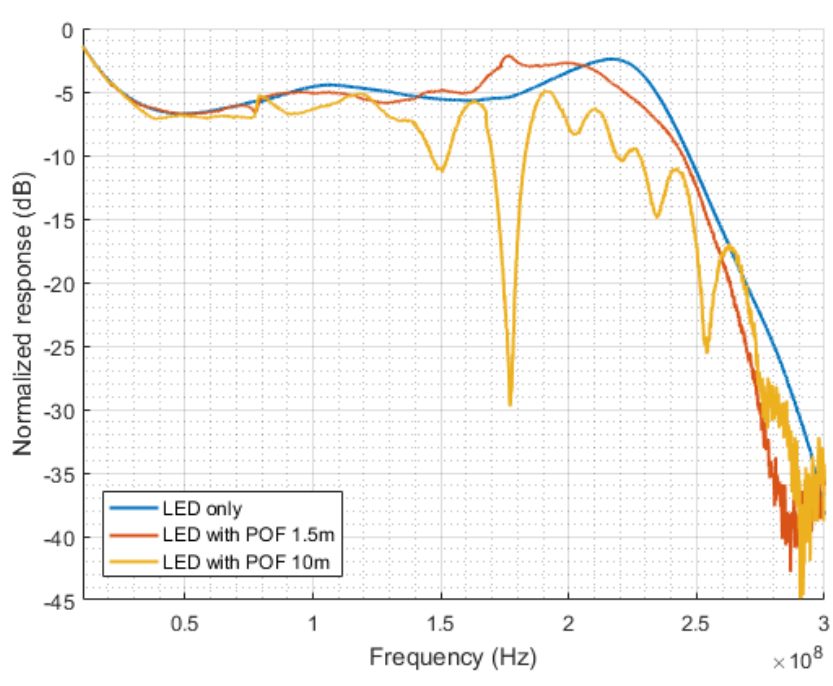

Fig. 6: Frequency response for the LED only and the LED including POF for various fiber lengths, all measured with a wideband reference photoreceiver in the lab. The response for $10 \mathrm{~m}$ POF connected to a first custom-designed POF receiver as shown in figure 3 .

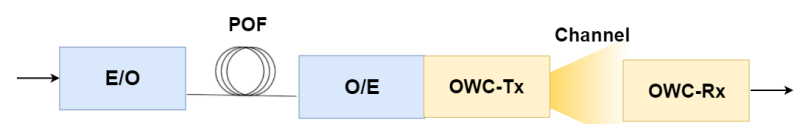

Fig. 7: Diagram of the combination of the POF and OWC links for further characterization. This corresponds to a SISO link of figure 2 .

\section{B. System-level evaluation}

Finally, the system-level performance is characterized. The performance of a communication link is limited by two main factors, bandwidth $B$ and signal-to-noise ratio $S N R$, as this is well known from channel capacity $C$ formulated by the Shannon-Hartley theorem for the capacity of a communication channel [15]. The highest achievable information rate that can be communicated without error is obtained with the well-known formula.

$$
C=B \cdot \log _{2}(1+S N R) b i t s / s
$$

As the SNR depends on frequency in the practical system, Equ.1 has to be applied at each frequency and averaged it. Complex modulation scheme such as OFDM can exploit a total system bandwidth which is typical higher than the $-3 \mathrm{~dB}$ bandwidth. Here we assume it can be better characterized by the $-10 \mathrm{~dB}$ bandwidth. Based on an assumed $S N R$ of $20 \mathrm{~dB}$, which is in practice limited by the wireless link, it is possible to estimate capacity by using equation 1 .

The result of capacity estimation for the individual POF and OWC links, as well as the POF+OWC link are shown in table I. Current results already indicate that, the capacity is well above $100 \mathrm{Mbit} / \mathrm{s}$ and fulfill the requirements for wireless communication in an industrial environment. 


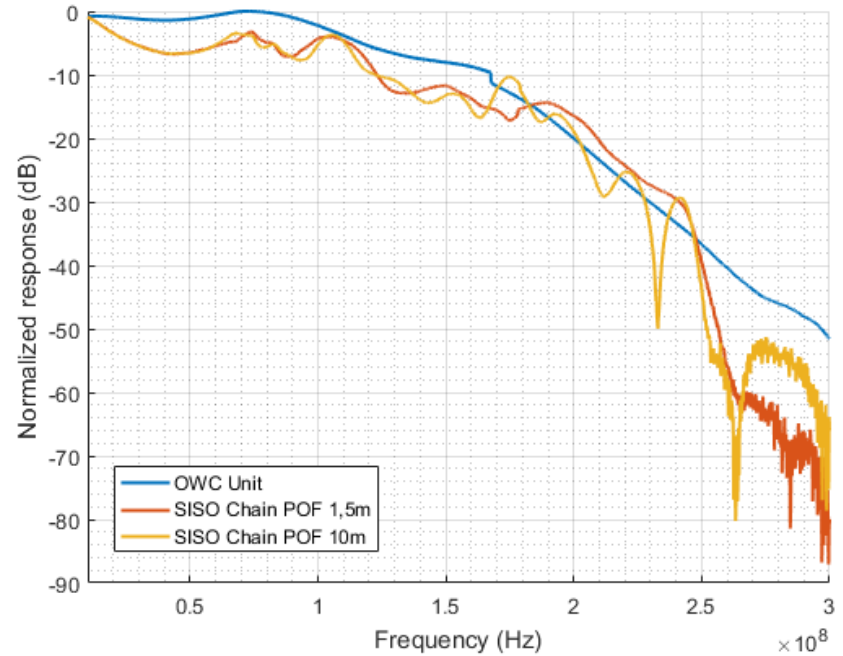

Fig. 8: Frequency response of the OWC unit alone and integrated with the POF link with various lengths.

TABLE I: Expected capacity according to equation 1.

\begin{tabular}{|c|c|c|}
\hline Link & Bandwidth $(-10 \mathrm{~dB})$ & Capacity \\
\hline POF $1.5 \mathrm{~m}$ & $244 \mathrm{MHz}$ & $1.07 \mathrm{Gbps}$ \\
\hline OWC & $167 \mathrm{MHz}$ & $733 \mathrm{Mbps}$ \\
\hline SISO chain with $10 \mathrm{~m} \mathrm{POF}$ & $122 \mathrm{MHz}$ & $535 \mathrm{Mbps}$ \\
\hline
\end{tabular}

\section{CONCLUSION AND Future Work}

In this paper, we have proposed and experimentally investigated the concept of using POF in distributed MIMO application suitable for industrial environment. This work was focused on designing the analog communication link based on POF and OWC link and, examining its main limitations. Experimental results showed that the requirement of industrial communication is already met by the distributed SISO link. Future work toward a D-MIMO system maximizing the reliability of communication using a large number of distributed optical front-ends will need the seamless integration of the POF link into the Li-Fi system. It will also be tested how much the overall SNR is degraded by the POF link, as operating at low SNR is another key requirement to be met in the industrial scenario.

\section{ACKNOWLEDGMENTS}

This work was supported in part by European project called VisIoN, funded by the European Union's Horizon 2020 research and innovation programme under the Marie Skłodowska-Curie grant agreement number 764461.

\section{REFERENCES}

[1] M. Uysal and H. Nouri, "Optical Wireless Communications - An Emerging Technology 1," Transparent Opt. Netw. 16th Int. Conf., pp. $1-7,2014$.

[2] D. Schulz et al., "Use cases for optical wireless communication," Opt. InfoBase Conf. Pap., vol. Part F84-O, pp. 1-3, 2018.

[3] S. Van Der Gaast, "Report by Stratix and TU/e," no. December, 2017.

[4] P. Wilke Berenguer et al., "Optical Wireless MIMO Experiments in an Industrial Environment," IEEE J. Sel. Areas Commun., vol. 36, no. 1, pp. 185-193, 2018.
[5] E. Biglieri, R. Calderbank, A. Constantinides, A. Goldsmith, A. Paulraj, and H. V. Poor, MIMO Wireless Communications. 2010.

[6] K. Wang, A. Nirmalathas, C. Lim, and E. Skafidas, "High speed $4 \mathrm{x}$ 12.5 Gbps WDM optical wireless communication systems for indoor applications," 2011 Opt. Fiber Commun. Expo., pp. 1-3, 2011.

[7] V. Jungnickel, "Optical Wireless in 5G Optical Wireless in 5G," no. August, 2016.

[8] L. Lovasz, "On the Shannon capacity of a graph," IEEE Trans. Inf. Theory, vol. 25, no. 1, pp. 1-7, Jan. 1979.

[9] X. Yang, F. Gao, J. Yan, and W. Shi, "Core Technology for Achieving Plastic Optical Fibers in an All-optical Network," vol. 3, no. 2, pp. 3-6, 2017.

[10] A. M. Zin, M. S. Bongsu, S. M. Idrus, and N. Zulkifli, "An Overview of Radio-over-Fiber Network Technology," pp. 0-2, 2010.

[11] J. Vinogradov, P. Optical, and F. Application, "Potential of High Speed, Short Distance Optical Data Communication on Large Diameter Optical Fibers," vol. 00, 2006.

[12] S. Loquai et al., "42-Gb / s Transmission Over Large-Core 1-mm PMMA Graded-Index Polymer Optical Fiber," vol. 25, no. 6, pp. 602-605, 2013.

[13] J. W. Wallenburg, "Polymer optical fibre: characterization and analog transmission," Graduation report, The Department of Electrical Engineering of the Eindhoven University of Technology, 1994.

[14] J. Kiermaier, "From SISO to MIMO - taking advantage of everything the air interface offers ( 2 )," News from Rohde Schwarz, vol. 194, no. 192, pp. 192-195, 2007.

[15] E. E. Narimanov and P. Mitra, "The Channel Capacity of a Fiber Optics Communication System: Perturbation Theory," vol. 20, no. 3, pp. 530-537, 2002. 